\title{
Preface to the "Special Issue on Science and Technologies for the Effective Use of Unrecovered Energy in Steelworks"
}

\author{
Tomohiro AKIYAMA \\ Center for Advanced Research of Energy and Materials, Faculty of Engineering, Hokkaido University, Kita 13 Nishi 8, Kita-ku, \\ Sapporo 060-8628 Japan.
}

The steelworks still have a large amount of outflowing unused energy, although several energy-recovery equipments such as coke dry quenching (CDQ) and top gas recovery turbine (TRT) have been widely applied. Since temperature of the outflowing heat from steelworks is relatively higher than the other industries and its amount reaches as much as $4 \%$ of primary energy consumed in Japan (see Fig. 1), their efficient recovery is urgently required to promote energy saving as one of the main countermeasures against global warming. According to the preliminary search, waste heat in steelworks is characterized by the following four points:

1) Most of the waste heat is emitted from batch processes.

2) The waste heat is usually recovered in the form of steam. However, long distance transportation of steam without heat loss is not possible.

3) Large thermal exergy in tar-containing coke oven gas, hot steel product and various molten slags have remained unrecovered because of technical difficulties in heat exchange.

4) Energy medium necessary to efficiently transport the recovered heat for long distance and to keep it for long time has not been developed.

In contrast, a worldwide attention has been recently focused on the 'latent heat storage' using PCM (Phase Change Material). For example, the 'TransHeat' container system has been proposed and applied in practice, which can repeatedly transport recovered heat in the industrial site for a municipal use by using PCM. In this case, the melting

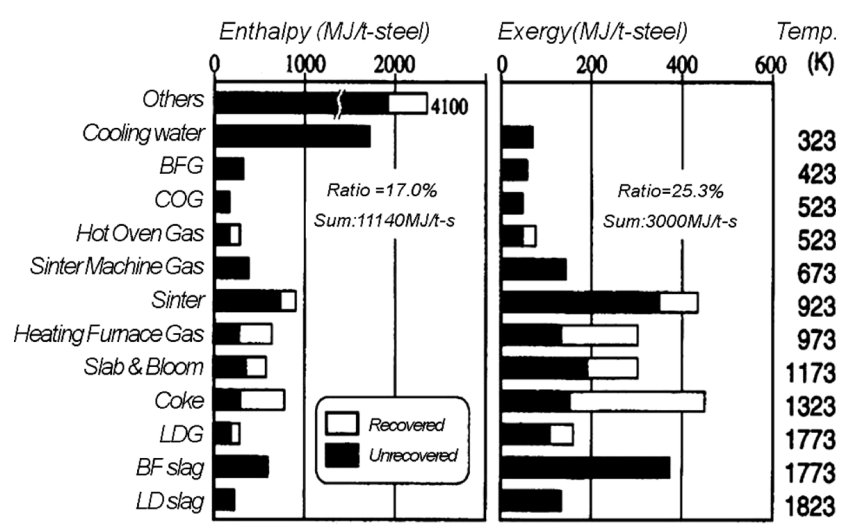

Fig. 1. point of PCM used is usually 58 or $118^{\circ} \mathrm{C}$.

In this aspect, a research project of "the Efficient Use of Waste Heat in the Steelworks" has been organized by 21 researchers with specialty of material science, chemical engineering and mechanical engineering as cooperative system between the universities and industries, and performed for four years (from 2006 to 2009FY). They mainly focused on the development of new PCM for high temperature application over $200^{\circ} \mathrm{C}$ in steelworks, and feasibility study on the combination of heat reuse process with waste heat using PCM. The cooperative project was successfully completed by five research groups. Their research subjects and the results were summarized as follows:

Group (I): Preliminary feasibility study on the optimum combination of energy accepter and energy donator from the viewpoints of amount, temperature level and time interval (Leader: Dr. T. Amagasa, JFE Steel).

Group (II): Development of new PCMs for high temperature applications, in which multiphase materials were systematically studied to control a melting point and latent heat. The results also demonstrated that alloys with large heat of solid-solid transformation and quenched slag can be practical PCM without encapsulation treatment (Leader: Prof. Dr. T. Nagasaka, Tohoku University).

Group (III): Design of PCM process, in which numerical/experimental simulation of fluid flow and heat transfer, supercooling of PCM, and transportation of reaction heat are mainly studied (Leader: Dr. T. Kurosaka, KOBELCO).

Group (IV): Process technologies for the utilization of waste heat such as rotary dryer using hot steel balls, thermoelectric device with PCM, steam electrolysis using PCM were proposed and verified as practical processes (Leader: Prof. Dr. E. Kasai, Tohoku University).

Group (V): New systems were examined based on the developed PCM technology through evaluation of financial benefit. In conclusion, several promising systems such as a combination of BTX production with waste heat of steel product were proposed and numerical target for the industrialization such as desired acceleration of heat exchange was declared (Leader: Dr. T. Nishimura, Nippon Steel Corporation).

The project report describing all the results obtained by the above research groups were distributed to the project 
members and related companies. On the basis of the above background, the papers of the members of the "Research Group on the Efficient Use of Waste Heat in the Steelworks" of ISIJ were invited to this special issue. Wide range of researches, which could be bases of new energysaving technologies/processes or a process improvements and also a technical developments of the process operation also contributed to the present issue. On behalf of the re- search project on the efficient use of waste heat in the steelworks, I am grateful to all the contributors of this issue.

It is our great pleasure that these papers are presented to the worldwide researchers in the fields of saving energy and reducing emission of greenhouse gas. We also hope the present issue is useful to many researchers to update the recent advances and to explore the prospective aspects in the technologies of energy saving in steelworks. 